\title{
Raman Spectroscopy Study of Pressure-Induced Structural Changes in Sodium Borate Glass
}

\author{
Mouritz N. Svenson ${ }^{1}$, Michael Guerette ${ }^{2}$, Liping Huang ${ }^{2}$, Morten M. Smedskjaer ${ }^{1, *}$ \\ ${ }^{1}$ Department of Chemistry and Bioscience, Aalborg University, 9220 Aalborg, Denmark \\ ${ }^{2}$ Department of Materials Science and Engineering, Rensselaer Polytechnic Institute, Troy, NY 12180, USA \\ * Corresponding author. e-mail: mos@bio.aau.dk
}

\begin{abstract}
Pressure-induced structural changes in a sodium borate glass have been studied up to $\sim 9$ GPa by in situ micro-Raman spectroscopy in a diamond anvil cell at ambient temperature. The results show a decrease in the fraction of boroxol rings and an increase in the fraction of non-ring structures with increasing pressure. These findings are compared with Raman spectra of the same glass composition compressed at $1 \mathrm{GPa}$ at its glass transition temperature $\left(406^{\circ} \mathrm{C}\right)$. We show similarities in the medium-range structural changes of the borate glass compressed at high-pressure/low-temperature $\left(9 \mathrm{GPa}, 25^{\circ} \mathrm{C}\right)$ and intermediate-pressure/hightemperature $\left(1 \mathrm{GPa}, \sim 400{ }^{\circ} \mathrm{C}\right)$ conditions. The structural changes are elastic during cold compression but inelastic as a result of the hot compression. We discuss this difference in relation to densification and Raman inactive structural changes.
\end{abstract}




\section{Introduction}

An efficient design of new glass materials requires a detailed understanding of their structure-property relations, which remains a key challenge within condensed matter physics. The macroscopic properties of glass depend on the network structure and connectivity, which in turn are a function of chemical composition and state variables such as temperature and pressure. Significant progress has been made in understanding structural variations and structure-property relations across varying compositions and temperatures at ambient pressure, whereas pressure-induced structural changes remain relatively poorly understood [1]. The glass structure can change gradually or abruptly during polyamorphic transition when an isostatic pressure is applied. Moreover, the modification of glass structure and properties depends on the utilized pressure route, e.g., static vs. dynamic compression [1] or compression in the rigid vs. non-rigid state $[2,3]$.

Densification of glass is often achieved by compression at room temperature (cold compression) in diamond anvil cells (DACs). Combined with in situ structural characterization (e.g., vibrational spectroscopy, inelastic x-ray scattering, $x$-ray and neutron diffraction), this has facilitated the exploration of key structural transformations occurring during densification within a variety of glass systems [4-11]. Using cold compression in DACs, high pressures above $10 \mathrm{GPa}$ can easily be reached, but only small sample specimens can be processed ( $\mu \mathrm{m}^{2}$ range). High pressures can also be reached in multianvil or piston cylinder devices, producing samples typically in the $\mathrm{mm}^{2}$ range. This is problematic in relation to analysis of macroscopic properties (e.g., hardness and durability) and for potential industrial applications, where larger sample specimens would typically be required.

Compression of glass at elevated temperature (hot compression) has been found to cause permanent densification at significantly lower pressures than those required in cold compression [12-16], with an accompanying change in the macroscopic properties [17-20]. At room temperature compression, most structural changes are reversible upon decompression at pressures below 5-10 GPa [1]. Hot compression enables the preparation of bulk sample specimens $\left(\mathrm{cm}^{2}\right.$ range), but while there have been numerous studies of glass structure as a function of pressure at room temperature, there are much fewer examples of the effects of simultaneous pressure and temperature treatment on glass structure $[2,13,16,21-22]$. The differences and 
similarities in the structural changes occurring during hot and cold compression are thus not well understood. Improving such understanding would provide information on the temperature-dependent densification mechanisms of disordered systems and potentially enable predictions of the structural changes occurring during hot compression based on results from cold compression experiments on analogous systems. This could in turn facilitate the development of glasses recovered from hot compression with tailored properties.

The structure of various archetypical glass-forming systems has been studied during cold compression, including vitreous $\mathrm{B}_{2} \mathrm{O}_{3}$. Borate glasses are interesting systems for high-pressure studies since they exhibit significant changes in both short- and intermediate-range order such as coordination number and fraction of superstructural units (boroxol groups), respectively. For example, compression of vitreous $\mathrm{B}_{2} \mathrm{O}_{3}$ leads to a breakage of boroxol rings, as supported by molecular dynamics simulations [23,24], Raman spectroscopy $[25,26],{ }^{11} \mathrm{~B}$ 3QMAS (triple quantum magic angle spinning) NMR (nuclear magnetic resonance) spectroscopy [27], ${ }^{11} \mathrm{~B}$ NMR spectroscopy [28], and x-ray diffraction results [28]. A polyamorphic phase transition has also been suggested to occur during decompression of vitreous $\mathrm{B}_{2} \mathrm{O}_{3}$ based on Brillouin scattering data [6,29-31] and molecular dynamics simulations [23].

When adding alkali oxides to $\mathrm{B}_{2} \mathrm{O}_{3}$ glass, non-monotonic changes in the properties occur since the network-modifying alkali ions can charge-stabilize boron in tetrahedral configuration [32,33]. However, only few high-pressure studies on alkali borate glasses have been conducted [4]. In this work, we investigate the structural changes during cold compression of a sodium borate glass using in situ Raman spectroscopy in a DAC. The observed structural modifications are discussed in relation to the changes occurring ex situ upon hot compression of the same glass composition. We emphasize the difference in elastic density increase (i.e., the recoverable deformation) and Raman inactive structural changes as the main difference between hot and cold compressed samples.

\section{Experimental procedure}

The nominal sample composition used for both cold and hot compression studies was $15 \mathrm{Na}_{2} \mathrm{O}-85 \mathrm{~B}_{2} \mathrm{O}_{3}$ (mol\%). The glass was produced from analytical grade $\mathrm{H}_{3} \mathrm{BO}_{3}$ and $\mathrm{Na}_{2} \mathrm{CO}_{3}$ powders, mixed according to the 
nominal composition and added stepwise to a $\mathrm{Pt}_{90} \mathrm{Rh}_{10}$ crucible at $1073 \mathrm{~K}$ for degassing. The mixture was then heated to $1273 \mathrm{~K}$ and kept for $1 \mathrm{~h}$. Afterwards it was heated to $1323 \mathrm{~K}$ and kept at this temperature for $15 \mathrm{~min}$ to ensure bubble removal before it was finally quenched to room temperature. The glass was annealed at its glass transition temperature $\left(T_{\mathrm{g}}=679 \mathrm{~K}\right)$ for $2 \mathrm{~h}$ to remove internal stress and then left overnight at $523 \mathrm{~K}$. To avoid hydration, which is a common problem for borate glasses [34], the glass was kept dry in desiccators with silica gel. The chemical composition was analyzed using wet chemistry method and found to be $15.4 \mathrm{Na}_{2} \mathrm{O}-84.6 \mathrm{~B}_{2} \mathrm{O}_{3}(\mathrm{~mol} \%)$.

Raman spectroscopic measurements were performed on a Horiba LabRAM HR800 spectrometer with an Olympus SLMPlan 50x objective lens with numerical aperture (NA) $=0.45$. A continuous wave $532 \mathrm{~nm}$ green laser was used as the probing light source. A membrane-driven DAC with diamond anvil culet size of $400 \mu \mathrm{m}$ was used for the cold compression experiment, with cryogenically loaded argon used as the pressure transmitting medium. The pressure inside the DAC was monitored from the fluorescent shift of ruby balls placed inside the sample chamber [35]. Following each step of pressure change in the DAC, an equilibration time of minimum $20 \mathrm{~min}$. was allowed before the Raman spectrum was collected. Several hours of equilibration time has previously been found to be required to achieve pressure equilibration in $\mathrm{B}_{2} \mathrm{O}_{3}$ glass [7], whereas this was not needed in the case of lithium borate glasses [36]. A general increase in background intensity was observed in the Raman spectra with increasing pressure inside the DAC. The Raman spectra were first processed by applying a background subtraction, using an asymmetric least squares baseline correction, followed by an area normalization procedure, where total area was transformed into constant area for all spectra [37]. All of the processed spectra were subsequently deconvoluted using Fityk software with Gaussian and Voigt lineshapes. Three separate deconvolutions were performed for each Raman spectrum. The deconvolution parameters presented in Figs. 3 to 5 represent average values from the three deconvolutions, with the error bars representing the standard deviations. An example of a deconvoluted Raman spectra is shown in Fig. 1. Due to fluorescence from the diamonds in the DAC, only the middle frequency region of the Raman spectra was analyzed $\left(350\right.$ to $\left.1000 \mathrm{~cm}^{-1}\right)$. 
Hot compression was peformed by isostatically compressing a sample at $1 \mathrm{GPa}$ at its ambient pressure $T_{\mathrm{g}}$ value in a vertically positioned gas pressure chamber, as described in detail elsewhere [17]. The general setup consists of a multizone cylindrical furnace, which is placed inside a gas pressure reactor with nitrogen as the compression medium. The samples were heated at a constant rate of $600 \mathrm{~K} / \mathrm{h}$ to $T_{\mathrm{g}}$ and the pressure was increased to $1 \mathrm{GPa}$. The samples were kept under these conditions for $30 \mathrm{~min}$ before the temperature was decreased to room temperature at a rate of $60 \mathrm{~K} / \mathrm{min}$, followed by decompression at a rate of 30 $\mathrm{MPa} / \mathrm{min}$. X-ray diffraction analysis showed no sign of crystallization. The glass transition temperature is a function of pressure [38], but the change is expected to be small within this pressure regime [39]. The Raman spectrum of the hot compressed sample was then collected ex situ as described above.

\section{Results and Discussion}

\subsection{Structural changes during compression in DAC}

In situ Raman spectra of the sodium borate glass during cold compression are shown in Fig. 2a. The most prominent features are the two sharp peaks around $800 \mathrm{~cm}^{-1}$, which are characteristic of alkali borate glasses with less than $20 \mathrm{~mol} \%$ alkali oxide [40-42]. The peaks in the spectra can be deconvoluted and assigned to the following structural units: $450 \mathrm{~cm}^{-1}$ (loose $\mathrm{BO}_{4}$ [43]), $650 \mathrm{~cm}^{-1}$ (loose $\mathrm{BO}_{3}$ [44]), $770 \mathrm{~cm}^{-1}$ (symmetric breathing vibration of triborate and/or tetraborate rings [45-48]), $805 \mathrm{~cm}^{-1}$ (symmetric breathing vibration of boroxol ring [49-53]), and $910 \mathrm{~cm}^{-1}$ (triborate and/or tetraborate [45,46]).

During compression, all Raman peaks decrease in intensity, except for the peak near $650 \mathrm{~cm}^{-1}$, which increases in intensity. Peak positions of the two sharp peaks at 770 and $805 \mathrm{~cm}^{-1}$ increase (Fig. 3), and the intensities decrease, approximately linearly with the applied pressure (Fig. 4). Complete suppression of the $805 \mathrm{~cm}^{-1}$ peak has previously been found to occur during cold compression of vitreous $\mathrm{B}_{2} \mathrm{O}_{3}$ at $11 \mathrm{GPa}$, in which case the peak position and intensity were also found to vary linearly with pressure [25]. The intensity of the $650 \mathrm{~cm}^{-1}$ peak increases approximately linearly with pressure (Fig. 5), whereas the position shifts towards lower frequencies (Fig. 2). This is surprising, since a shift towards higher frequencies is typically seen for Raman peaks during compression across a wide range of vibrational modes and compositions, 
including silicates [54-56], germinates [57,58], solid argon [59,60], and carbon based solids [61,62]. We should note that the intensities of the Raman peaks are relatively small compared to the background level, i.e., any subtle changes in background can cause changes in peak intensities. However, the background remains flat and uniform throughout the experiment with increasing pressure inside the DAC.

\subsection{Structural changes during decompression in DAC}

In situ Raman spectra of the sodium borate glass during decompression are shown in Fig. 2b. During decompression from $\sim 9 \mathrm{GPa}$, the peak positions follow the reverse route as during compression. To illustrate this, the deconvoluted positions of the two main peaks (around 770 and $805 \mathrm{~cm}^{-1}$ ) are shown in Fig. 3 as a function of pressure. A disconitnuity in the Raman and brillouin peak positions has previously been observed during decompression of vitreous $\mathrm{B}_{2} \mathrm{O}_{3}$ in the low pressure range $[6,25,29-31]$, but no discontinuity is found for the present sodium borate glass.

During decompression, the intensities and widths of the Raman peaks are reversible (i.e., elastic deformation), except for the peaks at 650 (loose $\mathrm{BO}_{3}$ ), 770 (triborate and/or tetraborate rings), and $805 \mathrm{~cm}^{-1}$ (boroxol rings). To illustrate this, the peak intensities of the $770 \mathrm{~cm}^{-1}$ and $805 \mathrm{~cm}^{-1}$ Raman peaks are shown as a function of pressure in Fig. 4, whereas the pressure dependence of the peak intensity of the $650 \mathrm{~cm}^{-1}$ peak is shown in Fig. 5. These peaks have been suggested to represent structural features involved in the network densification $[26,27,28,63]$, indicating that permanent densification occurs following cold compression of the sodium borate glass at $\sim 9 \mathrm{GPa}$. However, the pressure-induced density increase of cold compressed $\mathrm{B}_{2} \mathrm{O}_{3}$ glass (up to $9 \mathrm{GPa}$ ) has been found to relax over the course of days [64], i.e., the absent relaxation of the Raman peak intensities for the present sodium borate glass could be a result of slow structural recovery rather than a result of permanent densification.

\subsection{Densification mechanism during cold compression}

The pressure-induced decrease in the intensities of the Raman peaks representing ring structures $\left(770 \mathrm{~cm}^{-1}\right.$ and $805 \mathrm{~cm}^{-1}$ ) and the accompanying increase in the intensity of the Raman peak representing loose $\mathrm{BO}_{3}$

$\left(650 \mathrm{~cm}^{-1}\right)$ indicates a conversion of ring $\mathrm{BO}_{3}$ into non-ring $\mathrm{BO}_{3}$ during cold compression. The sodium borate 
glass is structurally analogous to $v-\mathrm{B}_{2} \mathrm{O}_{3}$ and is therefore expected to behave similarly under pressure and a breakage of boroxol rings has indeed been observed during cold compression of $v-\mathrm{B}_{2} \mathrm{O}_{3}$ by molecular dynamics simulations [23,24], neutron diffraction [64], and Raman spectroscopy [25] data. Furthermore, breakage of boroxol rings has also been found following hot compression of $\mathrm{v}-\mathrm{B}_{2} \mathrm{O}_{3}$ based on Raman spectroscopy [26], ${ }^{11} \mathrm{~B}$ 3QMAS NMR spectroscopy [27], ${ }^{11} \mathrm{~B}$ NMR spectroscopy [28], and x-ray diffraction [28] data. However, recently a reaction scheme involving the stacking of boroxol rings, rather than dissolution of the rings, has been suggested based on oxygen K-edge spectra of cold compressed v- $\mathrm{B}_{2} \mathrm{O}_{3}$ [7]. The reconfiguration of ring structures within the borate network under pressure is an ongoing research topic.

Compared to $v-\mathrm{B}_{2} \mathrm{O}_{3}$, less structural data are available for compression of alkali borate glasses. Based on the Raman spectra presented in this study, a conversion of rings into non-rings is considered to be the most likely scenario for the sodium borate glass. The conversion of rings into non-rings has been suggested as the main densification mechanism of $\mathrm{v}-\mathrm{B}_{2} \mathrm{O}_{3}[26-28,64]$, but we have recently found that even though this structural conversion is observed following hot compression of the sodium borate glass, it could not account for the density changes during subsequent ambient pressure annealing [65]. The major difference between the structural changes observed for $\mathrm{B}_{2} \mathrm{O}_{3}$ glass and those observed in the present $15.4 \mathrm{Na}_{2} \mathrm{O}-84.6 \mathrm{~B}_{2} \mathrm{O}_{3}$ glass is that in the $\mathrm{B}_{2} \mathrm{O}_{3}$ glass, only boroxol rings are converted into loose $\mathrm{BO}_{3}$ groups, whereas in the present sodium borate glass, triborate and/or tetraborate groups are also converted into loose $\mathrm{BO}_{3}$ groups. Changes in boron coordination are also expected at elevated pressure [36], but these have been found to be fully reversible during decompression [63], even if permanent densification is achieved [7].

\subsection{Comparison of cold and hot compression}

The conversion between ring and non-ring units discussed above is observed in situ during cold compression of the sodium borate glass and also following hot compression of the same glass composition at $1 \mathrm{GPa}$ at its glass transition temperature. Fig. 6 shows a comparison of the Raman spectra of the cold compressed (in situ) and hot compressed (ex situ) sodium borate glass. Here it can be seen that hot and cold compression causes the same qualitative changes in the Raman spectra. For example, an increase in the $650 \mathrm{~cm}^{-1}$ peak upon compaction of the glass. The spectrum shown for cold compression is taken at $1.4 \mathrm{GPa}$, i.e., within the 
elastic regime of densification. However, the pressure-induced structural changes are more pronounced than those found in the permanently densified hot compressed glass. This shows that the structural changes monitored by Raman spectroscopy can occur elastically during cold compression and in-elastically during hot compression.

In a recent study, we found that density changes in the sodium borate glass after hot compression and during subsequent ambient pressure annealing (volume relaxation) were not governed by structural changes detectable by Raman spectroscopy [65]. This indicates that the densification mechanism is Raman inactive. Other structural probes may, however, be capable of monitoring Raman inactive structural changes, displaying the differences in structural changes occurring following hot compression (permanent densification) and during cold compression (elastic densification). For example, a recent study on a boroaluminosilicate glass has showed a decrease in the ${ }^{11} \mathrm{~B}$ MAS shift after hot compression, whereas an increase in the ${ }^{11} \mathrm{~B}$ NMR shift has been observed in situ upon cold compression [63]. The decrease of the ${ }^{11} \mathrm{~B}$ NMR shift upon hot compression and increase upon cold compression in the boroaluminosilicate glass indicates that fundamentally different structural changes occur during hot and cold compression. Differences in the structural changes during hot and cold compression have also recently been found for vitreous $\mathrm{SiO}_{2}$, i.e., hot and cold compressed $\mathrm{SiO}_{2}$ glasses with similar densities have different network structures [66]. Moreover, cold compression has been found to result in a more heterogeneous glass structure than hot compression [16].

A conversion of rings into non-rings is evident from the Raman spectra during both hot and cold compression of the sodium borate glass (Fig. 6). That is, the Raman peaks found to exhibit intensity increase (decrease) following hot compression also exhibit intensity increase (decrease) during cold compression. However, Raman inactive structural changes may behave differently during hot and cold compression, as recently found for a boroaluminosilicate glass [63]. The conversion between ring and non-ring $\mathrm{BO}_{3}$ is expected to require the breakage of chemical bonds, but occurs elastically during cold compression and inelastically during hot compression. This behavior could be explained if the conversion between ring and non-ring $\mathrm{BO}_{3}$ is a byproduct of increased density, whether transient or permanent. However, density increase is governed by Raman inactive structural changes. That is, if the Raman inactive structural changes behave 
elastically during cold compression, the density would recover and consequently also the related Raman active structural changes. On the other hand, if the Raman inactive structural changes are permanent following hot compression, densification and Raman active structural changes would also both be permanent.

\section{Conclusion}

We have performed in situ Raman spectroscopy measurements on a sodium borate glass at pressures up to $\sim 9 \mathrm{GPa}$ at room temperature. With increasing pressure, a decrease in the fractions of six membered triborate, tetraborate, and boroxol rings has been found to occur concurrent with an increase in the fraction of non-ring $\mathrm{BO}_{3}$ groups. These pressure-induced structural changes have been compared with those from ex situ Raman spectroscopy measurements on the same sodium borate glass, which has been subjected to compression at 1 GPa at its glass transition temperature. Qualitatively the same changes are identified in the Raman spectra as a result of both cold and hot compression and our results imply that Raman inactive structural changes govern pressure-induced densification. This may explain how the same changes in the Raman spectra can occur elastically during cold compression and inelastically during hot compression, since these structural changes may be governed by density, which in turn is governed by Raman inactive structural changes.

\section{Acknowledgements}

M.N.S. and M.M.S. acknowledge financial support from the Danish Council for Independent Research under Sapere Aude: DFF-Starting Grant (1335-00051A). M.G and L.H. acknowledge financial support from the US National Science Foundation under Grant No. DMR-0907076 and DMR-1255378. 


\section{References}

1. P. Salmon, A. Zeidler, Networks under pressure: the development of in situ high-pressure neutron diffraction for glassy and liquid materials, J. Phys.: Condens. Matter 27 (2015) 133201

2. J.D. Mackenzie, High-Pressure Effects on Oxide Glasses: I, Densification in Rigid State, J. Am. Ceram. Soc. 46 (1963) 461-470

3. J.D. Mackenzie, High-pressure Effects on Oxide Glasses: III, Densification in Nonrigid State, J. Am. Ceram. Soc . 47 (1964) $76-80$

4. S.K. Lee, P.J. Eng, H-k. Mao, J. Shu, Probing and modeling of pressure-induced coordination transformation in borate glasses: Inelastic x-ray scattering study at high pressure, Phys. Rev. B. 78 (2008) 214203

5. S. Buchner, A.S. Pereira, J.C. de Lima, N.M. Balzaretti, X-ray study of lithium disilicate glass: High pressure densificationand polyamorphism, J. Non-Cryst. Solids 387 (2014) 112-116

6. J. Nicholas, S. Sinogeikin, J. Kieffer, J. Bass, Spectroscopic Evidence of Polymorphism in Vitreous $\mathrm{B}_{2} \mathrm{O}_{3}$, Phys. Rev.Lett. 92 (2004) 215701

7. S. Lee, P. Eng, H-k. Mao, Y. Meng, M. Newville, M.Y. Hu, J. Shu, Probing of bonding changes in $\mathrm{B}_{2} \mathrm{O}_{3}$ glasses at high pressure with inelastic X-ray scattering, Nat. Mat. 4 (2005) 851-854

8. C. Meade, R. Hemley, H. Mao, High-pressure x-ray diffraction of $\mathrm{SiO}_{2}$ glass, Phys. Rev. Lett. 69 (1992) 1387

9. C.J. Benmore, E. Soignard, S.A. Amin, M. Guthrie, S.D. Shastri, P.L. Lee, J.L. Yarger, Structural and topological changes in silica glass at pressure, Phys. Rev. B. 81 (2010) 054105

10. S. Susman, K.J. Volin, D.L. Price, M. Grimsditch, J.P. Rino, Intermediate-range order in permanently densified vitreous $\mathrm{SiO}_{2}$ : A neutron-diffraction and molecular-dynamics study, Phys.

Rev. B. 43 (1991) 1194

11. F. Galeener, A. Leadbetter, M. Stringfellow, Comparison of the neutron, Raman, and infrared vibrational spectra of vitreous $\mathrm{SiO} 2, \mathrm{GeO} 2$, and BeF2, Phys. Rev. B. 27 (1983) 1052

12. I.M. Kanzaki, Melting of Silica up to 7 GPa, J. Am. Ceram. Soc. 73 (1990) 3706 
13. B. Poe, C. Romano, G. Henderson, Raman and XANES spectroscopy of permanently densified vitreous silica, J. Non-Cryst. Solids 341 (2004) 162-169

14. T. Deschamps, J. Margueritat, C. Martinet, A. Mermet, B. Champagnon, Elastic Moduli of Permanently Densified Silica Glasses, Sci. Rep. 4 (2014) 7193

15. D. Uhlmann, Densification of alkali silicate glasses at high pressure, J. Non-Cryst. Solids 13 (1973) 89-99

16. M. Guerette, M.R. Ackerson, J. Thomas, F. Yuan, E.B. Watson, D. Walker, L. Huang, Structure and Properties of Silica Glass Densified in Cold Compression and Hot Compression, Sci. Rep. 5 (2015) 15343

17. M.M. Smedskjaer, S. Rzoska, M. Bockowski, J.C. Mauro, Mixed alkaline earth effect in the compressibility of aluminosilicate glasses, J. Chem. Phys. 140 (2014) 054511

18. M.M. Smedskjaer, R.E. Youngman, S. Striepe, M. Potuzak, U. Bauer, J. Deubener, H. Behrens, J.C. Mauro, Y.Z. Yue, Irreversibility of Pressure Induced Boron Speciation Change in Glass Sci. Rep. 4 (2014) 3770

19. M.N. Svenson, T. Bechgaard, S. Fuglsang, R. Pedersen, A. Tjell, M. Østergaard, R.E. Youngman, J.C. Mauro, S.J. Rzoska, M. Bockowski, M.M. Smedskjaer, Composition-Structure-Property Relations of Compressed Borosilicate Glasses, Phys. Rev. Appl. 2 (2014) 024006

20. S. Striepe, M.M. Smedskjaer, J. Deubener, U. Bauer, H. Behrens, M. Potuzak, R.E. Youngman, J.C. Mauro, Y.Z. Yue, Elastic and micromechanical properties of isostatically compressed soda-limeborate glasses, J. Non-Cryst. Solids 364 (2013) 44-52

21. K.J. Arndt, D. Stoffler, Anomalous changes in some properties of silica glass densified at very high pressures, Phys. Chem. Glas. 10 (1969) 117

22. S. Hofler, F. Seiferst, Volume Relaxation of Compacted $\mathrm{SiO}_{2}$ Glass - a Model for the Conservation of Natural Diaplectic Glasses, Earth Planet. Sci. Lett. 67 (1984) 433-438

23. L. Huang, J. Nicholas, J. Kieffer, J. Bass, Polyamorphic transitions in vitreous $\mathrm{B}_{2} \mathrm{O}_{3}$ under pressure, J.Phys.: Condens. Matter 20 (2008) 075107 
24. A Zeidler, K. Wezka, D.A.J. Whittaker, P.S. Salmon, A. Baroni, S. Klotz, H.E. Fischer, M.C. Wilding, C.L. Bull, M.G. Tucker, M. Salanne, G. Ferlat, M. Micoulaut, Density-driven structural transformations in $\mathrm{B}_{2} \mathrm{O}_{3}$ glass, Phys. Rev. B 90 (2014) 024206

25. M. Grimsditch, A. Polian, A.C. Wright, Irreversible structural changes in vitreous $\mathrm{B}_{2} \mathrm{O}_{3}$ under pressure, Phys. Rev. B. 54 (1996) 152

26. S. Sharma, B. Simons, J. Mammone, Relationship between density, refractive index and structure of $\mathrm{B}_{2} \mathrm{O}_{3}$ glasses at low and high pressures, J. Non-Cryst. Solids 42 (1980) 607-618

27. S.K. Lee, K. Mibe, Y. Fei, G.D. Cody, B.O. Mysen, Structure of $\mathrm{B}_{2} \mathrm{O}_{3}$ Glass at High Pressure: A $\mathrm{B}^{11}$ Solid-State NMR Study, Phys. Rev.Lett. 94 (2005) 165507

28. V.V. Brazhkin, I. Farnan, K.I. Funakoshi, M. Kanzaki, Y. Katayama, A. Lyapin, H. Saitoh, Structural Transformations and Anomalous Viscosity in the $\mathrm{B}_{2} \mathrm{O}_{3}$ Melt under High Pressure, Phys. Rev. Lett. 105 (2010) 115701

29. J. Nicholas, R.E. Youngman, S. Sinogeikin, J. Bass, J. Kieffer, Structural changes in vitreous boron oxide, Phys. Chem. Glasses 44 (2003) 249-251

30. M. Grimsditch, R. Bhadra, Brillouin scattering from amorphous materials at high pressures, Phys. Rev. B. 38 (1988) 7836

31. J. Nicholas, S. Sinogeikin, J. Kieffer, J. Bass, A high pressure Brillouin scattering study of vitreous boron oxide up to 57 GPa J. Non-Cryst. Solids 3492004 30-34

32. P. Bray, J. O'Keefe, Nuclear magnetic resonance investigations of the structure of alkali borate glasses, Phys. Chem. Glasses 4 (1963) 37

33. M. Hubert, A.J. Faber, On the structural role of boron in borosilicate glasses, Phys. Chem. Glasses: Eur. J. Glass Sci. Technol B 55 (2014) 136-158

34. T. Rockett, W. Foster, Phase Relations in the System Boron Oxide-Silica, J. Am. Ceram. Soc. 48 (1965) $75-80$

35. F. Datchi, A. Dewaele, P. Loubeyre, R. Letoullec, Y. Le Godec, B. Canny, Optical pressure sensors for high-pressure-high-temperature studies in a diamond anvil cell, High Pressure Res. 27 (2007) $447-463$ 
36. S. Lee, P. Eng, H-K. Mao, Y. Meng, J. Shu, Structure of Alkali Borate Glasses at High Pressure: B and Li K-Edge Inelastic X-Ray Scattering Study Phys. Rev. Lett. 98 (2007) 105502

37. J. Felten, H. Hall, J. Jaumot, R. Tauler, A. Juan, A. Gorzsás, Vibrational spectroscopic image analysis of biological material using multivariate curve resolution-alternating least squares (MCRALS), Nat. Prot.10 (2015) 217-240

38. A. Drozd-Rzoska, S.J.Rzoska, M. Paluch, A.R. Imre, C.M. Roland, On the glass temperature under extreme pressures, J. Chem. Phys. 126 (2007) 164504

39. N.S. Bagdassarov, J. Maumus, B. Poe, A. Slutskiy, V.K. Bulatov, Pressure dependence of $T_{g}$ in silicate glasses from electrical impedance measurements Phys. Chem. Glasses 45 (2004) 197-214

40. T. W. Bril, Raman spectroscopy of crystalline and vitreous borates, Ph.D. thesis, Technische Hogeschool, Eindhoven, 1976.

41. W. Konijnendijk, J. Stevels, The structure of borate glasses studied by Raman scattering, J. NonCryst. Solids 18 (1975) 307-331

42. T. Yano, N. Kunimine, S. Shibata, M. Yamane, Structural investigation of sodium borate glasses and melts by Raman spectroscopy.: I. Quantitative evaluation of structural units, J. Non-Cryst. Solids $321(2003) 137$

43. E. Kamitsos, M. Karakassides, G. Chryssikos,Structure of borate glasses. Part 1. Raman study of caesium, rubidium, and potassium borate glasses,Phys. Chem. Glasses 30 (1989) 229

44. G. Walrafen, S. Samanta, P. Krishnan, Raman investigation of vitreous and molten boric oxide J. Chem. Phys. 72 (1980) 113

45. B. Meera, J. Ramakrishna, Raman spectral studies of borate glasses J. Non-Cryst. Solids 159 (1993) $1-21$

46. A. Wright, C. Vedishcheva, Superstructural unit species in vitreous and crystalline alkali, alkaline earth and related borates Phys. Chem. Glasses: Eur. J. Glass Sci. Technol B 54 (2013) 147-156

47. W. Konijnendijk, J. Stevels, The structure of borate glasses studied by Raman scattering J. NonCryst. Solids 18 (1975) 307-331 
48. R.N. Sinclair, R. Haworth, A.C. Wright, B. G. Parkinson, D. Holland, J. W. Taylor, N. M. Vedishceva, I. G. Polyakova, B. A. Shakhmatkin, S. A. Feller, B. Rijal, T. Edwards, Neutron Spectroscopic Studies of Caesium Borate Crystals and Glasses, Phys. Chem. Glasses 47 (2006) 405-411

49. G. Ellison, L. Panek, P. Bray, G. Rouse, Determinations of structure and bonding in vitreous $\mathrm{B}_{2} \mathrm{O}_{3}$ by means of $\mathrm{B}^{10}, \mathrm{~B}^{11}$, and $\mathrm{O}^{17} \mathrm{NMR}$, J. Chem. Phys. 66 (1977) 802

50. R. Mozzi, B. Warren, The structure of vitreous boron oxide, J. Appl. Cryst. 3 (1970) 251-257

51. C.F. Windisch, W.M. Risen, Raman spectroscopic study of molecular orientation in vitreous $\mathrm{B}_{2} \mathrm{O}_{3}$ films, J. Non-Cryst.Solids 48 (1982) 325-344

52. C.F. Windisch, W.M. Risen, Vibrational spectra of oxygen- and boron-isotopically substituted $\mathrm{B}_{2} \mathrm{O}_{3}$ glasses, J. Non-Cryst. Solids 48 (1982) 307-323

53. G. Ferlat, T. Charpentier, A.P. Seitsonen, A. Takada, M. Lazzeri, L. Cormier, G. Calas, F. Mauri, Boroxol Rings in Liquid and Vitreous $\mathrm{B}_{2} \mathrm{O}_{3}$ from First Principles Phys. Rev. Lett. 101 (2008) 065504

54. T. Deschamps, C. Martinet, J. Bruneel, B. Champagnon, Soda-lime silicate glass under hydrostatic pressure and indentation: a micro-Raman study J. Phys.: Condens. Matter 23 (2011) 035402

55. B. Mysen, J. Frantz, Silicate melts at magmatic temperatures: in-situ structure determination to $1651^{\circ} \mathrm{C}$ and effect of temperature and bulk composition on the mixing behavior of structural units, Contrib. Mineral. Petr. 117 (1994) 1-14

56. R. Hemley, H. Mao, P. Bell, B. Mysen, Raman Spectroscopy of $\mathrm{SiO}_{2}$ Glass at High Pressure, Phys. Rev. Lett. 57 (1986) 747

57. R. Le Parc, V. Ranieri, J. Haines, M. Cambon, O. Cambon, C. Levelut, S. Clément, In situ high pressure and high temperature Raman studies of $(1-\mathrm{x}) \mathrm{SiO}_{2} \mathrm{xGeO}$ glasses, J. Phys.: Condens. Matter 21 (2009) 375109

58. S. Sharma, T. Cooney, Z. Wang, S. van der Laan, Raman band assignments of silicate and germanate glasses using high-pressure and high-temperature spectral data, J. Raman. Spectrosc. 28 (1997) 697-709 
59. G. Watson, W. Daniels, Raman scattering from solid argon at high pressure Phys. Rev. B 37 (1988) 2629

60. R. Crawford, D. Bruns, D. Gallagher, M. Klein, Raman scattering from condensed argon, Phys. Rev. B 17 (1978) 4871

61. J. Sandler, M. Shaffer, A.Windle, M.P. Halsall, M. A. Montes-Morán, C.A. Cooper, R.J. Young, Variations in the Raman peak shift as a function of hydrostatic pressure for various carbon nanostructures: A simple geometric effect Phys. Rev. B 67 (2003) 035417

62. H. Boppart, J. van Straaten, I. Silvera, Raman spectra of diamond at high pressures, Phys. Rev. B $32(1985) 1423$

63. T. Edwards, T. Endo, J. Walton, S. Sen, Observation of the transition state for pressure-induced BO3 $\rightarrow$ BO4 conversion in glass, Science 345 (2014) 1027-1029

64. A. Wright, C. Stone, R. Sinclair, Structure of pressure compacted vitreous boron oxide Phys.Chem. Glasses 41 (2000) 296

65. M.N. Svenson, M.M. Smedskjaer, Y.Z. Yue, R.E. Youngman, S.J. Rzoska, M. Bockowski, L.R. Jensen, Structural Relaxation of Isostatically Compressed Sodium-Borate Glass. In: ACers GOMDDGG Joint Annual Meeting; 2015 May 17-21; Miami, FL, abstract no. GOMD-S3-165-2015.

66. C. Martinet, A. Kassir-Bodon, T. Dechamps, A. Cornet, S. Le Floch, V. Martinez, B. Champagnon, Permanently densified $\mathrm{SiO}_{2}$ glasses: a structural approach, J. Phys.: Condens. Matter 27 (2015) 325401 


\section{FIGURES}

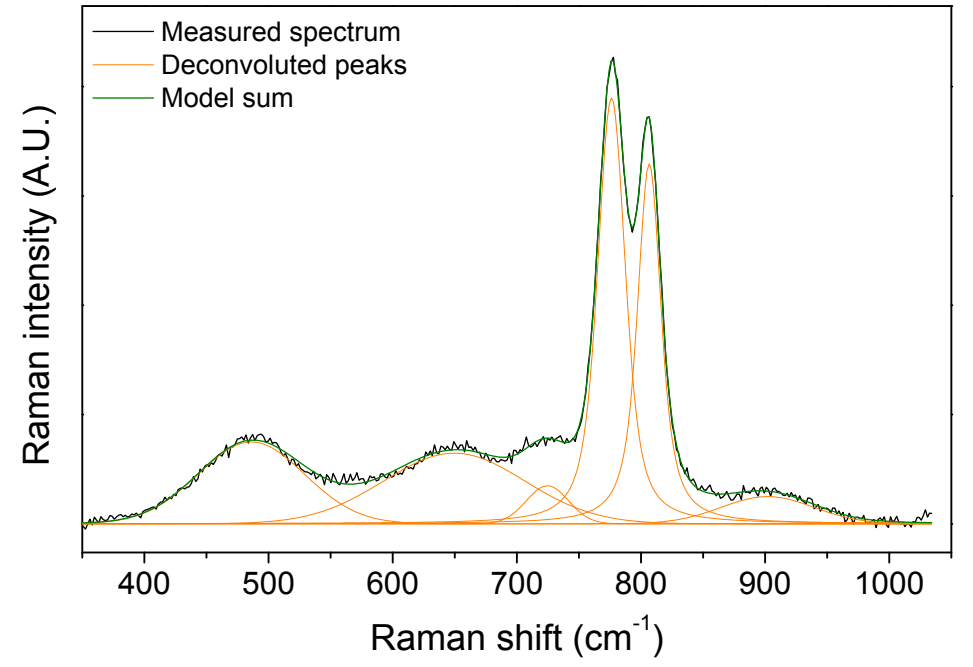

Figure 1. Example of deconvoluted Raman spectrum sodium borate glass in situ at $1.4 \mathrm{GPa}$. Voigt lineshapes are used to fit the two main bands $\left(770\right.$ and $\left.805 \mathrm{~cm}^{-1}\right)$, whereas Gaussian lineshapes are used for the remaining bands. 

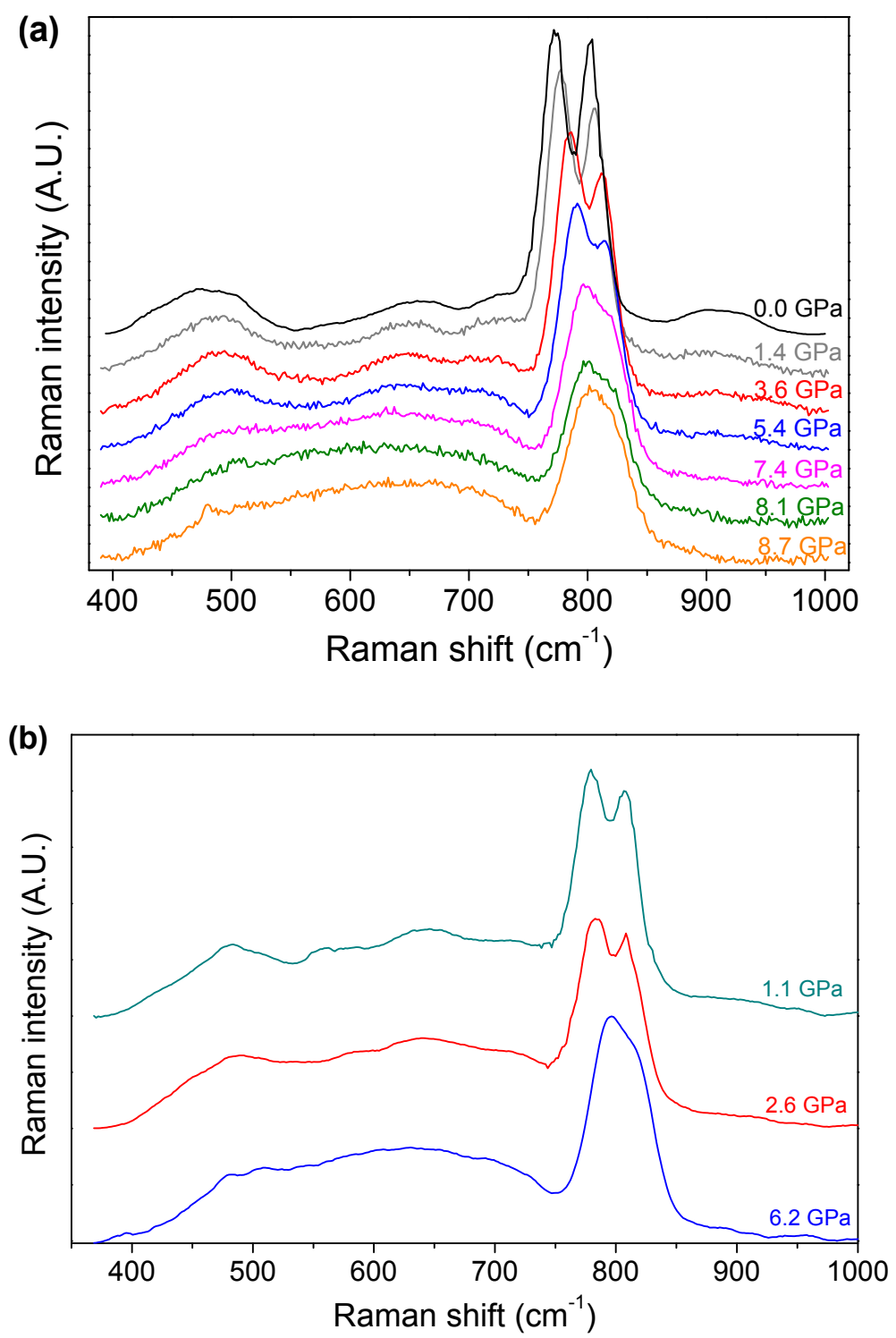

Figure 2. In situ micro-Raman spectra of sodium borate glass during (a) cold compression up to $\sim 9 \mathrm{GPa}$ and (b) subsequent decompression. Spectra are shifted vertically for clarity. Major changes in peak intensities and positions are seen for the two main peaks (around 770 and $805 \mathrm{~cm}^{-1}$ ) as well as for the $650 \mathrm{~cm}^{-1}$ peak. Peaks gradually recover during decompression. A mild smoothing algoritm has been applied to the spectra of the as-prepared sample and those acquired during decompression due to their relatively high noise-to-signal ratio. 


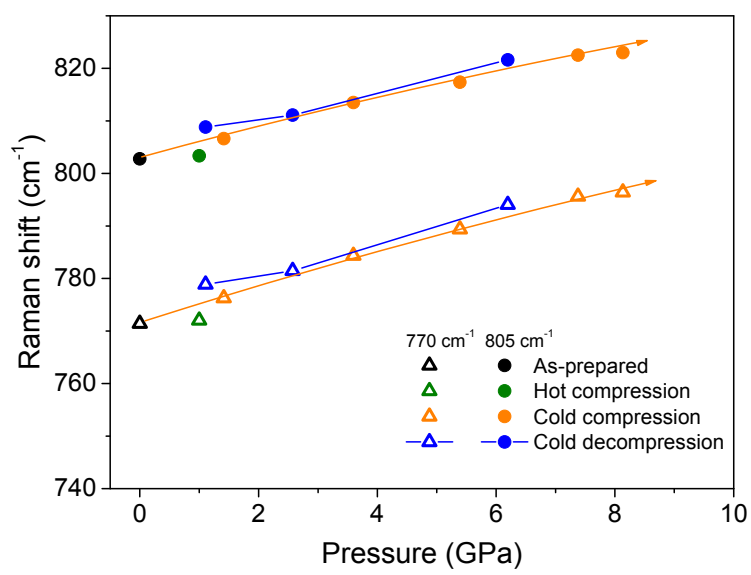

Figure 3. Positions of the two main Raman peaks (around 770 and $805 \mathrm{~cm}^{-1}$ ) during in situ cold compression and ex situ after hot compression at $1 \mathrm{GPa}$. Positions of Raman peaks in the as-prepared glass is included for comparison. A monotonic increase in the peak positions with pressure is found for both peaks during cold compression, with an elastic recovery during decompression. The lines are guides for the eye. Error bars are smaller than the size of the symbols. 


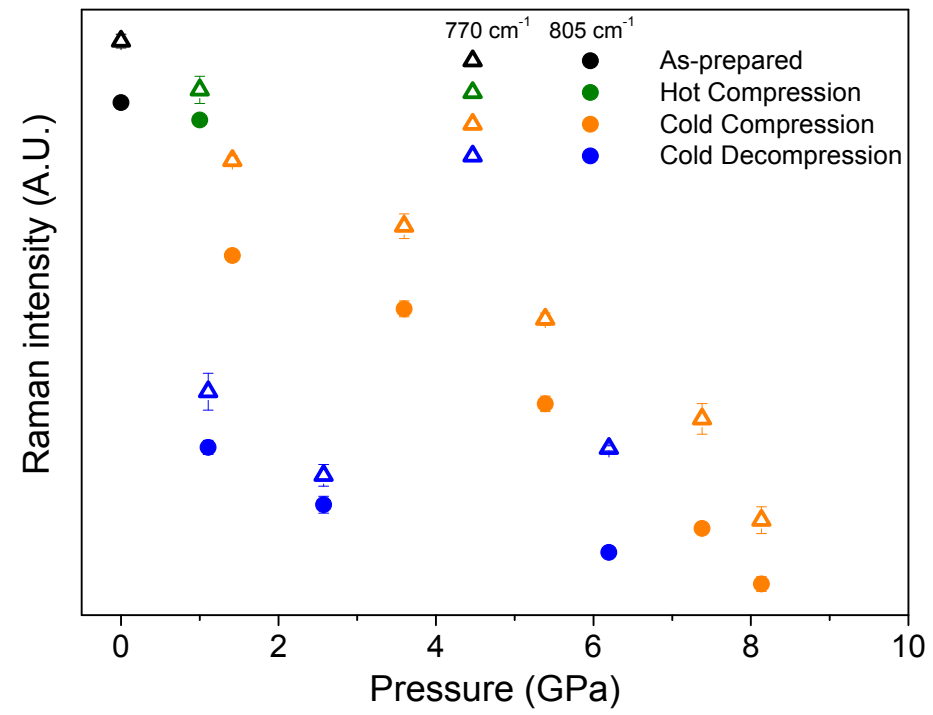

Figure 4. Intensities of the two main Raman peaks near 770 and $805 \mathrm{~cm}^{-1}$ during in situ cold compression and ex situ after hot compression at $1 \mathrm{GPa}$. The results of the as-prepared glass are included for comparison. Error bars respresent standard deviations for three individual deconvolutions of the Raman spectra. 


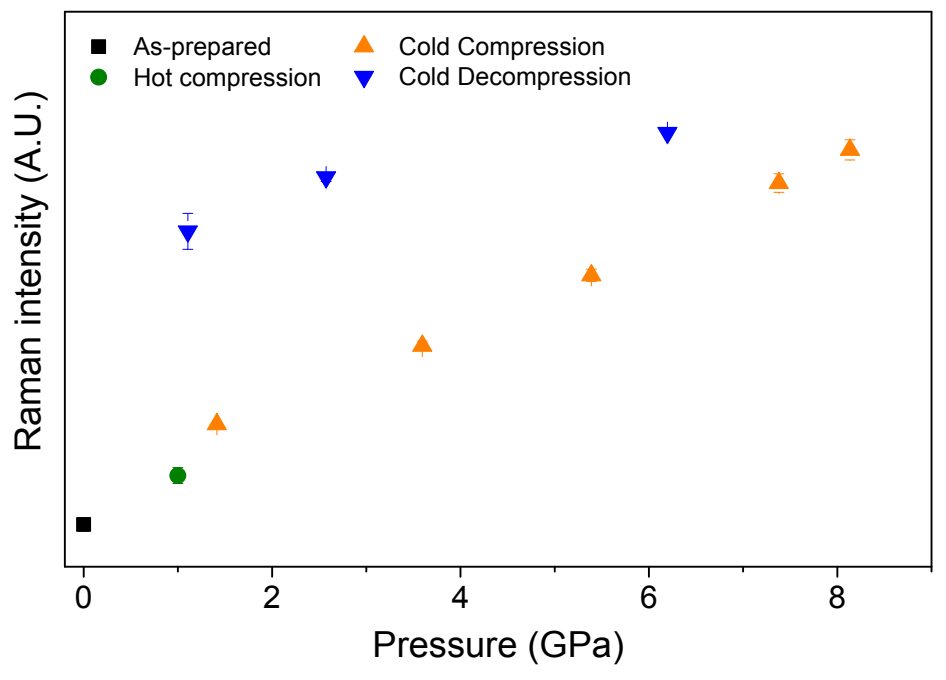

Figure 5. Intensity of the $650 \mathrm{~cm}^{-1}$ Raman peak during in situ cold compression and ex situ after hot compression at $1 \mathrm{GPa}$. The result of the as-prepared glass is included for comparison. Error bars represent standard deviations for three individual deconvolutions of the Raman spectra. 


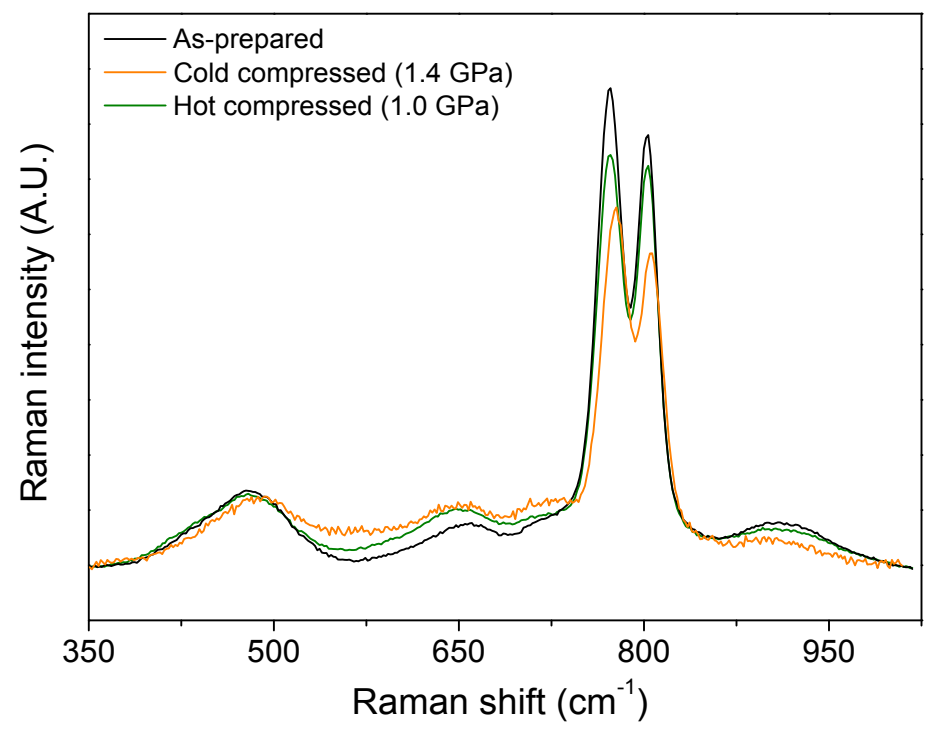

Figure 6. Comparison of Raman spectrum of the as-prepared sodium borate glass with that of the same glass subjected to hot compression and an in situ spectrum collected during cold compression at a pressure similar to that of the hot compressed sample (1.0 and $1.4 \mathrm{GPa}$, respectively). All of the peaks exhibit changes in intensites upon compression (except the $450 \mathrm{~cm}^{-1}$ peak), including shifts in the peak positions. 